\title{
Enhanced energy transfer efficiency in a four-electrodes configuration DBD plasma jet
}

\author{
Fellype do Nascimento ${ }^{1, a}$, Munemasa Machida ${ }^{2}$, Konstantin Kostov ${ }^{3}$, Stanislav Moshkalev ${ }^{1}$, \\ Roberto Y. Honda ${ }^{3}$, Rogério P. Mota ${ }^{3}$, Thalita M.C. Nishime ${ }^{3}$, and Alonso H.R. Castro ${ }^{3}$ \\ ${ }^{1}$ Center for Semiconducting Components and Nanotechnologies - State University of Campinas, Campinas, Brazil \\ 2 Institute of Physics "Gleb Wataghin" - State University of Campinas, Campinas, Brazil \\ ${ }^{3}$ Faculty of Engineering - Sao Paulo State University, Guaratinguetá, Sao Paulo, Brazil
}

Received 26 May 2017 / Received in final form 8 August 2017

Published online 7 November 2017 - (C) EDP Sciences, Società Italiana di Fisica, Springer-Verlag 2017

\begin{abstract}
In this work a dielectric barrier discharge (DBD) plasma jet that uses a multiple electrodes configuration is investigated. The results show that both plasma power and its rotational and vibrational temperatures tend to increase with the number of powered electrodes in the DBD device. The emission intensities of the excited species in the plasma, and consequently their number density, also grow as a function of the number of powered electrodes. Based on these facts and since the electric power provided by the power supply was kept constant, there is an indication that the use of multiple electrodes improves the energy efficiency of the device.
\end{abstract}

\section{Introduction}

Atmospheric pressure plasma jets (APPJs) are kind of gas discharges that can be operated at near ambient pressure and temperature. In these devices barrier discharge is usually excited in a noble gas that flows through a thin dielectric tube and the resulting plasma is projected into the surrounding ambient forming a several cm-long plasma plume. Although gas temperature of the plume can be kept close to room temperature, energetic plasma species (electrons and metastables) interact with air molecules producing different reactive species, such as, reactive oxygen (ROS) and nitrogen (RNS) species, neutrals in fundamental and excited states and energetic photons. In the last decade, cold APPJs have attracted significant attention due to their simple construction, great versatility, low cost and broad range of applications spanning from material processing [1] to medicine [2] and dentistry [3]. A detailed overview of these devices, their excitation schemes and electrode configurations as well as perspectives for future development can be found in a comprehensive review article [4].

Since APPJs generate plasma plumes in open space they can be easily adapted to treat irregular 3D objects and also implemented in in-line industrial processing. However, in most excitation schemes and working gases the plasma plume size is normally limited by the inner diameter of the employed dielectric tube [5]. This feature is advantageous in cases when a localized treatment is needed, like in dentistry and microelectronics, where ultrafine capillaries with aperture of $0.1-1.0 \mu \mathrm{m}$ are used [6].

\footnotetext{
${ }^{\mathrm{a}}$ e-mail: fellype@gmail.com
}

When plasma jet hits a target the plasma spreads on the surface covering an area that can be more than twice the jet cross section [7]. Moreover, long-living active species produced in the plasma can diffuse radially outward forming reactive zone around the plasma column where the material surface properties will be modified [8,9]. However, in the most cases the target area modified by the plasma jet ranges from few $\mathrm{mm}^{2}$ to about $1 \mathrm{~cm}^{2}[7-9]$.

Many different applications require a plasma jet with large cross-section however building such device with uniform plasma distribution is quite a challenging task. First of all, simply increasing the diameter of dielectric tube is not a solution because it leads to excessively high gas flows and also intense electric fields are required for plasma ignition. Different geometry of the tube exit, like a tapered or horn-like nozzle investigated in [10], affects the length and size of produced plasma jet. The authors in [11] obtained a 2-cm-diam plasma jet by using three (a powered, a grounded and a floated) electrodes. Cooperative merging of two or four He plasma jets into one large plasma column ( $\sim 2$-cm-diam) was reported in [12] and [13], respectively. However, only within a limited range of gas flows and applied voltages it was possible to form one uniform plasma plume. Another approach suitable for large area applications is building a close-packed array of many parallel plasma jets [14]. The jets are combined into a honeycomb $[15,16]$ or linear $[17,18]$ structures and normally they share the same gas feed and power supply. However, as reported by some authors [18] the individual jets interact with each other (hydro-dynamically by their gas flows and electrically by their electric charges) thus making difficult to ignite all plasma jets in a uniform manner. Usually some jets from the array are more intense and 
longer than others [15] and also plasma plumes bending due to jet-jet interaction was observed $[16,18]$.

In this work we report a new plasma jet configuration with four high-voltage electrodes covered by dielectric barriers that can generate a stable plasma jet with $\sim 0.2$ $0.8 \mathrm{~cm}^{2}$ cross-section using same electric power as the one provided by the power supply in one jet configuration. The main feature to obtain such a large plasma diameter configuration is to work on during the initial stage of surface plasma formation at the gas input region. In the quadrupole configuration the intense electric fields produced by high voltage electrodes helps forming one larger plasma ignition region instead of separated jet, and therefore avoiding jet-jet interaction or plasma plume bending.

\section{Experimental setup}

The schematic of the multi-electrode DBD device is shown in Figure 1. It consists of a dielectric tube in which are inserted four 2.5-mm-diam. copper rods encapsulated in closed-end glass tubes. Some of the main device dimensions are: inner and outer diameter of the outer dielectric tube equal to 17.5 and $32 \mathrm{~mm}$, respectively and outer diameter of the glass tubes is $4.3 \mathrm{~mm}$. The electrodes are arranged in a square with sides equal to $7.0 \mathrm{~mm}$. The length of the glass tubes that penetrates inside the outer dielectric tube is $50 \mathrm{~mm}$. The main dielectric enclosure terminates with an exchangeable nozzle that can have different opening. In order to produce a plasma jet, a continuous gas flow is injected inside the outer dielectric tube and an alternate high-voltage is applied to one or more electrodes. Also, if necessary, some metal rods can be removed from the glass enclosures leaving a reduced number of powered electrodes. When the discharge is ignited the plasma is concentrated around the glass tubes and more specifically in the central region between the four glass tubes, where a stable plasma column is formed. Then a plasma plume is extracted into the air in direction of a grounded metal disc with radius $5 \mathrm{~cm}$ that is covered by a 12-cm-diam ceramic plate. The distance between the ceramic plate and exit nozzle was kept $5 \mathrm{~mm}$. In this work we use two nozzles with different aperture diameters of $5 \mathrm{~mm}$ (to be called "small nozzle" from now on) or $10 \mathrm{~mm}$ in diameter (to be called "big nozzle" from now on).

The gas flow rate was fixed at 1.9 liters per minute. We used He as the working gas with a purity of $99.998 \%$ in all experiments reported in this work, except in cases where the use of another gas is mentioned. The device was operated in an open environment, that is, at atmospheric pressure and with the ambient gas surrounding the plasma jet.

To obtain the emission spectra of the plasmas in this work we used a multichannel Avantes spectrometer (model AvaSpec-ULS2048X64T), equipped with a 600 lines/mm grating. The light emitted by the plasma jets were collected with a cosine lens, with $10 \mathrm{~mm}$ in diameter and placed $10.0 \mathrm{~cm}$ from the center of the plasma column, thus giving us a collection area with dimensions of approximately $10.0 \mathrm{~mm} \times 5.0 \mathrm{~mm}$, integrated along a

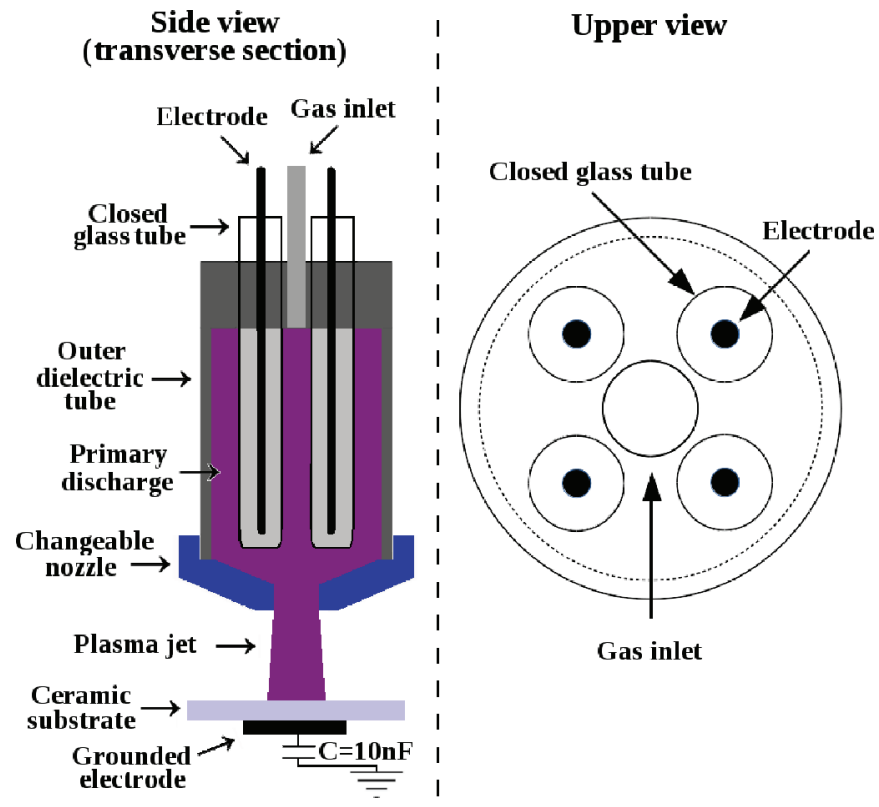

Fig. 1. Schematic of the 4-electrodes DBD reactor. The elements are out of scale.

line of sight through the plasma column. After that, the light was transported to the spectrometer through an optical fiber. The spectra were acquired in a continuous mode using an integration time of $9.69 \mathrm{~ms}$ in all cases. The aperture of the entrance slit of the spectrometer was $25 \mu \mathrm{m}$, giving us a spectral resolution of $(0.67 \pm 0.02)$ $\mathrm{nm}$. A relative calibration of intensity of the spectrometer was performed in order to verify that there is no significant variation (less than 1\%) in the sensitivity within the narrow wavelength range $(\sim 25 \mathrm{~nm})$ used to calculate the rotational and vibrational temperatures $\left(T_{\text {rot }}\right.$ and $T_{v i b}$, respectively).

The plasma power calculations were made using a well-known method $[19,20]$ measuring simultaneously the voltage $(V)$ applied to the electrode(s) and the charge transferred to a capacitor that is in series to the grounded stage. We obtain the value of the plasma power by measuring the area of the Q-V Lissajous figure and multiplying it by the frequency $(f)$ of the applied voltage signal:

$$
P=f \oint V d Q .
$$

For all power calculations we used a sinusoidal highvoltage signal, produced by a Minipuls4 power supply (GBS Elektronik GmbH, Germany), operating with fixed amplitude and frequency of $20 \mathrm{kV}$ peak-to-peak and $10 \mathrm{kHz}$, respectively. The voltage measurement on the electrode(s) and also the operating frequency, were monitored using a high-voltage Tektronix probe P6015A and an oscilloscope (Tektronix TDS 3032, $300 \mathrm{MHz}$ ). The Miniplus4 power supply is very sensitive to network matching, so that both the voltage and the operating frequency could change with the number of electrodes in the DBD device. In order to circumvent this issue a fast response high voltage capacitor $(C=1 \mathrm{nF})$ was connected in parallel with 


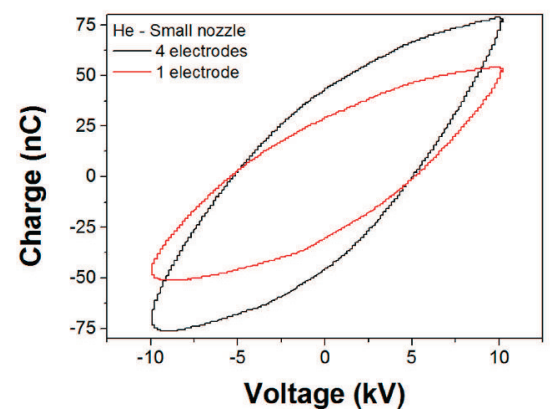

(a)

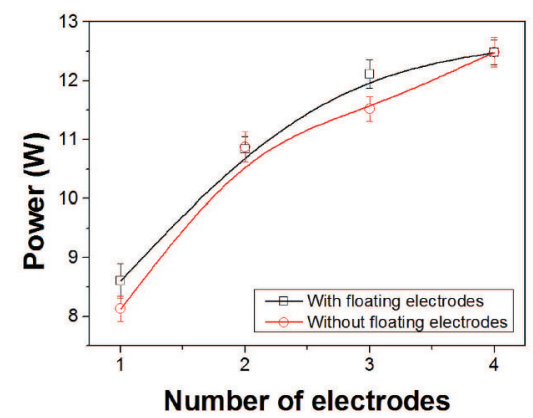

(b)

(b)

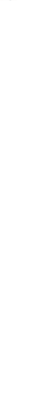

Fig. 2. Q-V Lissajous figures for 1 and 4 electrodes (a) and power vs. number of powered electrodes (b) using the small nozzle.

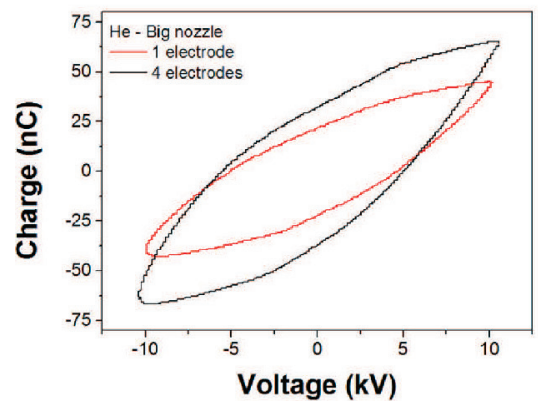

(a)

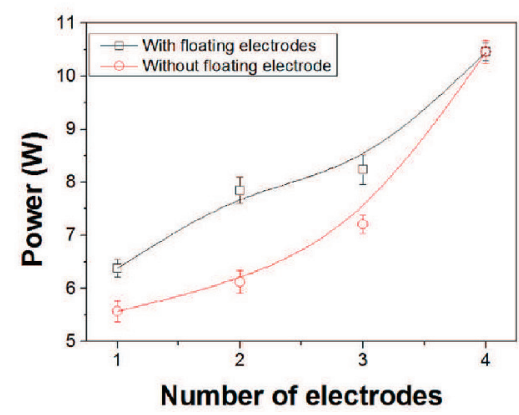

(b)

Fig. 3. Q-V Lissajous figures for 1 and 4 electrodes (a) and power vs. number of powered electrodes (b) using the big nozzle.

the high voltage output and this configuration allowed the experiments to be performed keeping constant both the voltage amplitude and frequency.

\section{Results and discussion}

The characterization of the multi-electrode DBD device made in this work consists of measurement of important plasma parameters like plasma power, rotational and vibrational temperatures, and identification of emitting species present in the plasma.

In order to obtain the dependence of plasma parameters as a function of the number of electrodes we started with four powered electrodes in the DBD device and this number was being reduced one by one until only one active electrode remained. There are two conditions for power measurements with less than four electrodes: the first one is simply to disconnect electrically each electrode, maintaining it in the device, and then it will act as a floating electrode. And the second one is to remove each electrode from the device for the subsequent measurement. We performed the measurements first using the DBD device with the small nozzle and, after that, the power measurements were repeated using the big nozzle. In all cases when we had only two powered electrodes they were arranged in opposite corners of the device. For rotational and vibrational temperature measurements as a function of the number of electrodes a similar procedure was adopted, but the temperatures were measured only for the small nozzle and in the floating electrodes condition.

\subsection{Power measurements}

Figure 2 shows Q-V Lissajous figures (a) and curves of power as a function of the number of powered electrodes (b) operating with the small nozzle. The Q-V figures shown in Figure 2a were obtained for four electrodes and for one electrode with the other three in the floating condition.

As we can see from the area of the $\mathrm{Q}-\mathrm{V}$ figures in Figure 2, the energy deposited in the plasma, and, consequently, the power, increases significantly ( $\sim 45-55 \%)$ when we switch from one to four electrodes in the device. Furthermore, the growth of the plasma power with the number of electrodes is monotonic, both with and without floating electrode, indicating certain proportionality between these parameters. Figure $2 \mathrm{~b}$ shows that the presence of floating electrodes inside the DBD device with small exit nozzle does not affect significantly the discharge power. Therefore, optical emission spectra were taken at these conditions with the floating electrodes.

The results for power measurements using the big nozzle are shown in Figure 3. The Q-V Lissajous figures (a) and curves of power as a function of the number of electrodes (b) also show that the energy deposited in the plasma and its power increase as a function of the number of electrodes when the big nozzle is used. In this case, the increment in the power when switching from one to four electrodes is about $\sim 65-90 \%$ and is higher than in the small nozzle case. However, the absolute value of power is lower in the big nozzle case.

It is important to stress that the voltage applied to the electrodes was kept constant in the power supply and did 
not varied significantly (less than $5 \%$ ) when the number of electrodes was changed. With this in mind and observing Figures 2 and 3, we verify that the use of multiple electrodes in a DBD device leads to a good improvement on its energy efficiency. Since the addition of powered electrodes to the DBD device changes the capacitance and resistance of the system, it could lead to an impedance matching which in turn could be the reason for that improvement in the energy efficiency. In order to discover if it occurs, we used the multi-electrode device in combination with another power supply, which operates in the pulsed mode [21], and measured the electric current signals that pass through the plasma and across a shunt resistor, placed similarly to the capacitor shown in Figure 1, using 1 to 4 electrodes applying a voltage equals to $15 \mathrm{kV}$ in all cases. This power supply is able to deliver always the same amount of energy in each pulse, since the electric energy it can supply is that stored in a set of capacitors subjected to the working voltage. The gas used in this case was argon. These measurements are shown in Figure 4, where we can see that the peak values of the electric current measured also increase when the number of powered electrodes increase. In Figure 4 we also can notice that the width of the electric current peaks are higher with the increase in the number of powered electrodes. Since the voltage pulses applied to the electrodes did not changed significantly among the measurements, we can consider that the variations in current values and their widths are directly proportional to the respective power variations and this means that the power increased with the number of electrodes even in the case where a pulsed power source was used. Then we can discard any relationship between the impedance matching with the energy transfer efficiency.

For the device with big nozzle the lack of floating electrodes resulted in about $20 \%$ power reduction but in both cases the power increases monotonically with the number of powered electrodes. It is also important to notice that the symmetry does not play an essential role in the increment of the plasma power with the number of electrodes. We can state this based on the fact that in our tests (not shown in this paper) power measurements made using two electrodes arranged in opposite or adjacent corners of the square in the device did not presented significant differences. Nevertheless the variation of the distance between the electrodes should play important role in the electric field distribution, affecting therefore the power efficiency in the big or small nozzle case.

One of the advantages to have a DBD plasma with a higher power is that it may improve the surface modification of polymers $[22,23]$, reducing the contact angle of plasma processed surfaces with the increase of plasma power. Another advantage is that higher values of plasma power leads to more electron energy (electron temperature) and it may favor the production of reactive radicals (like $\mathrm{NO}$ and $\mathrm{OH}$ ) in the plasma.

Some possible explanations for the plasma power growth with addition of powered electrodes to the DBD device may be related to the increment in the surface charges accumulated at the surfaces of the inner dielectric tubes that is in contact with the primary plasma.

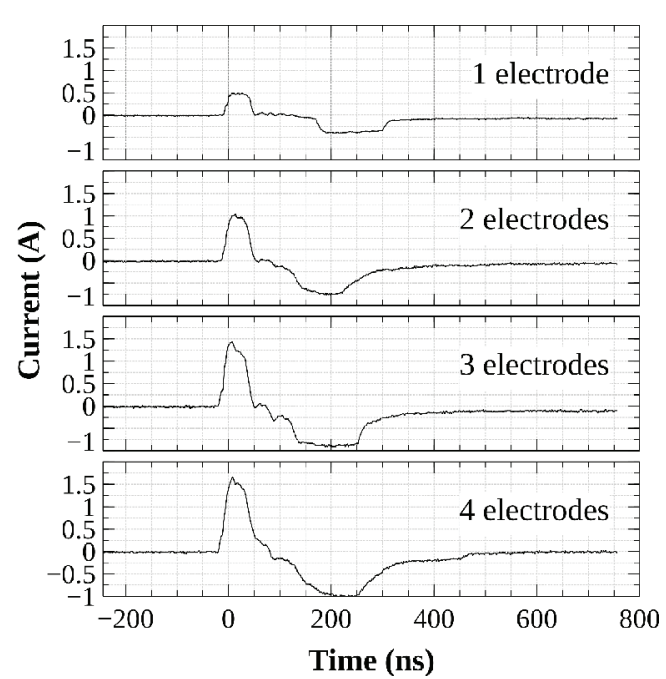

Fig. 4. Electric current signals obtained using a pulsed power supply [21] combined with the multi-electrode device using 1 to 4 electrodes.

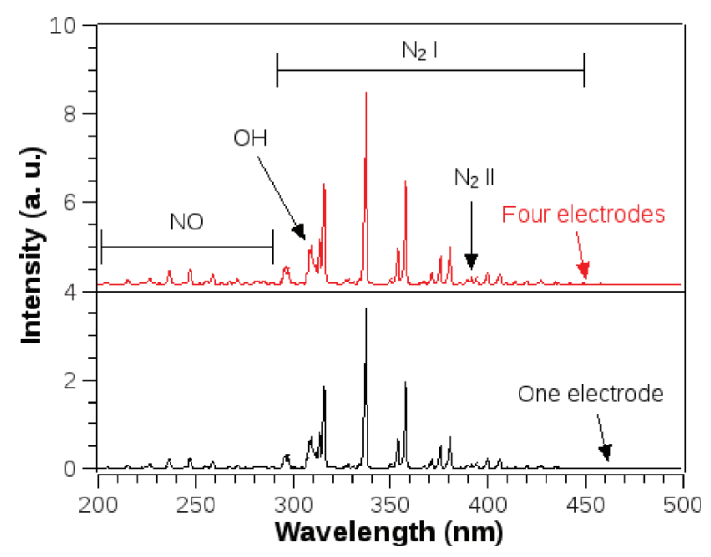

Fig. 5. Optical emission spectra obtained in DBD discharges using the small nozzle using 1 electrode (below) and 4 electrodes (above).

Also, as more electrodes are added both surface charges and their electric fields are summed up leading to increase of the total electric field. With the increase of the surface charges the propagation of surface ionization waves are facilitated and the increase of the electric field accelerates that waves [24-26].

\subsection{Optical characterization}

In order to identify the emitting species present in the plasma we measured the optical emission spectrum of the plasma jet in the wavelength range from 200 to $500 \mathrm{~nm}$. Figure 5 shows the spectra obtained for DBD discharges using one electrode (below) and four electrodes (above), and Figure 6 shows the variation of the emission intensities as a function of the number of powered electrodes for select lines from nitric oxide (NO) and neutral $\mathrm{N}_{2}\left(\mathrm{~N}_{2} \mathrm{I}\right)$ in Figure $6 \mathrm{a}$, and from hydroxide $(\mathrm{OH})$ and ionized $\mathrm{N}_{2}\left(\mathrm{~N}_{2} \mathrm{II}\right)$ in Figure 6b. The data presented in Figures 5 and 6 were taken in the floating electrodes condition with the small nozzle. 

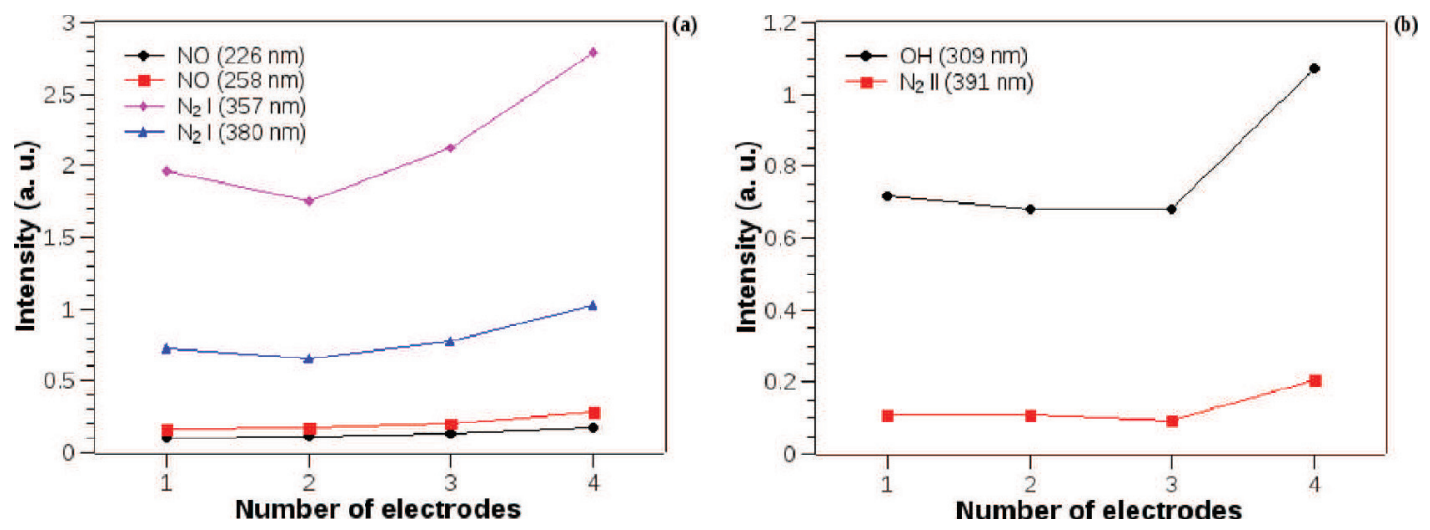

Fig. 6. Variation of the intensity as a function of the number of powered electrodes for some selected emissions using the small nozzle in the floating electrodes condition.

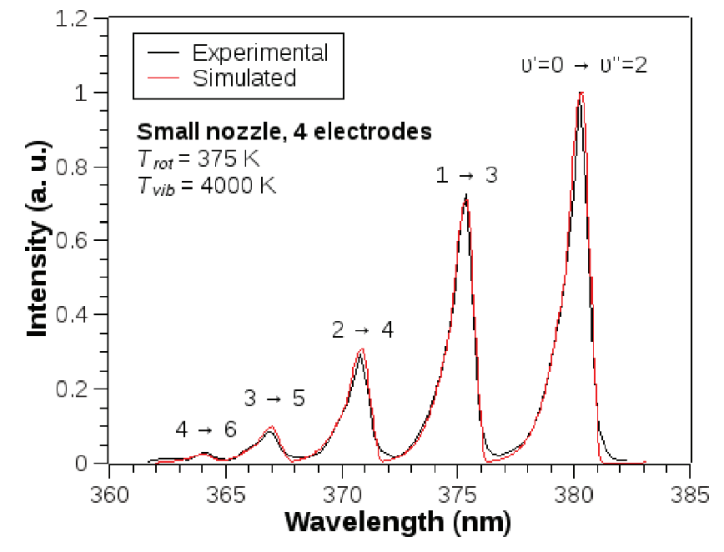

Fig. 7. Characteristic curves for determination of $T_{\text {rot }}$ and $T_{v i b}$ : experimental and simulated curves for $\mathrm{N}_{2}$ I molecular emission band ( $\mathrm{C}^{3} \Pi_{u}, \nu^{\prime} \rightarrow \mathrm{B}^{3} \Pi_{g}, \nu^{\prime \prime}$ transitions) obtained using the small nozzle with 4 electrodes. The intensity of the emissions are normalized to 1 at the highest peak.

In the spectra shown in Figure 5 we have found strong emissions from $\mathrm{NO}$ radical in the ultra-violet region between 200 and $290 \mathrm{~nm}$, besides more commonly observed $\mathrm{OH}$ radical near $309 \mathrm{~nm}$, and $\mathrm{N}_{2}$ I from 295 to $470 \mathrm{~nm}$, or emission from $\mathrm{N}_{2}$ II at $391.44 \mathrm{~nm}$. There are no detectable He emission lines in this spectral region.

As can be seem comparing the spectra in Figure 5, regarding the species found in the plasma, there are no significant differences between the emission spectra obtained when the number of electrodes is changed.

In relation to the intensity as a function of the number of electrodes, we noticed that it tends to increase when the number on electrodes changes from one to four for all emitting species, but the growth is monotonic only for $\mathrm{NO}$ emission lines. The curves shown in Figure 6a for NO and $\mathrm{N}_{2}$ I show that the behavior of the intensity as a function of the number of powered electrodes does not depend strongly on the wavelength of the emissions, being similar for the same species in all wavelengths in which it emits. Since the plasma power increases with the number of powered electrodes, as was verified in the previous section, and the emission intensities also do so, we can say that the emission intensities, which are proportional to the number density of its respective emitting specie, also grow as a function of the plasma power.
In order to obtain $T_{\text {rot }}$ and $T_{v i b}$ we measured the emission spectrum of nitrogen molecules. The transitions used for the temperature calculations were those from the second positive system $\left(\mathrm{N}_{2} \mathrm{I}, \mathrm{C}{ }^{3} \Pi_{u}, \nu^{\prime} \rightarrow \mathrm{B}^{3} \Pi_{g}, \nu^{\prime \prime}\right)$ in the wavelength range from 360 to $385 \mathrm{~nm}$. Then, the measured spectra were compared with simulated ones using the SpecAir software [27] for various values of $T_{\text {rot }}$ and $T_{v i b}$ until we find the values that best fit the simulated curves to the experimental ones. This method is well stablished and is very well accepted by the academic community for obtaining of $T_{\text {rot }}$ and $T_{v i b}$ [28-30]. The second positive system of $\mathrm{N}_{2}$ in the selected wavelength range is one of the most used to measure vibrational temperatures in non-thermal plasmas due to the high number of transitions that can be observed (up to 5, depending on the vibrational temperature achieved by the plasma). With more transitions available, the determination of rotational temperatures, which affect the shape and width of the observed transitions, are also benefited and becomes more accurate. In the simulations we assumed a triangular slit function with a basis equal to $1.36 \mathrm{~nm}$. Figure 7 shows an example of experimental and simulated curves used to obtain the temperatures.

In Figure 8, two important plasma parameters, the rotational and vibrational temperatures, are shown as a function of the number of powered electrodes. As we can see in Figure 8, both $T_{\text {rot }}$ and $T_{v i b}$ tend to increase with the number of powered electrodes in the DBD device, but the increment in $T_{\text {rot }}$ does not exceed the uncertainty of the measurements, thus the $T_{\text {rot }}$ values remain low (about $350 \pm 25 \mathrm{~K}$ ), which is desirable for treatment of temperature sensitive materials like biological tissues. On the other hand, the $T_{v i b}$ values increase a little bit more (about $200 \mathrm{~K}$ ) when we switch from one to four electrodes. The highest $T_{v i b}$ value $(4100 \pm 50 \mathrm{~K})$ obtained in the multielectrode DBD device is one of the highest reported for a DBD discharge produced at atmospheric pressure in open environment.

The increment in the $T_{v i b}$ values is a good result since a recent study [31] has indicated that plasmas with higher values of $T_{v i b}$ keeping about the same $T_{\text {rot }}$ could be better to perform surface treatment of materials, improving their wettability and reducing the treatment time needed to achieve the desired results. 


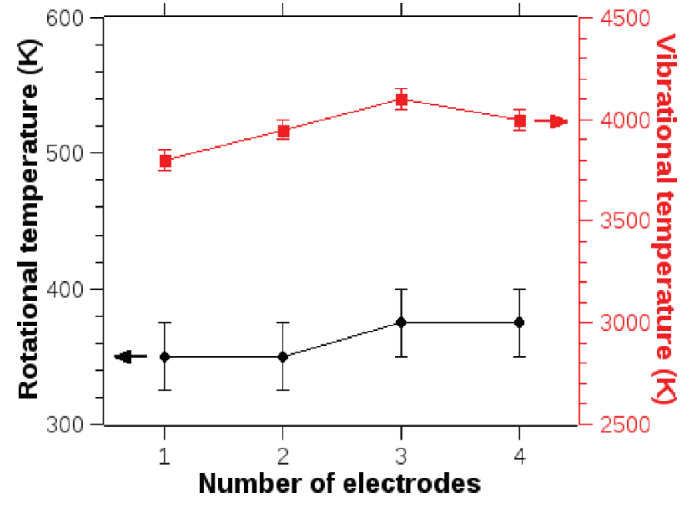

Fig. 8. Rotational and vibrational temperatures as a function of the number of electrodes in the multi-electrode DBD device obtained using the small nozzle with floating electrodes.

\section{Conclusions}

One of the main findings of this work is that it is possible to deliver more energy to a plasma in a DBD device just increasing the number of powered electrodes without changing any other parameter in the power supply. In other words, we found good evidences that there is a way to improve the energy efficiency of a plasma source. It was verified measuring the plasma power, the emission intensities and the temperatures $T_{\text {rot }}$ and $T_{v i b}$, and noticing that all of them tend to increase as a function of the number of powered electrodes in the device.

Since both the plasma power and its vibrational temperature are parameters that may improve the results on surface treatment of materials, and both are parameters whose values increase with the number of powered electrodes in the DBD device, we can infer that a way to optimize such devices for surface treatment is to find an adequate number of electrodes and a configuration that maximize the product power $\times T_{v i b}$.

Further studies using other geometric parameters, configurations, number of electrodes, and also the use of other gases are being conducted in order to provide a better understanding of the energy efficiency improvement with the variation of the number of powered electrodes in the device.

This work was supported by $\mathrm{CNPq}$ (processes number 150570/2016-3) and FAPESP under Grant 2015/21989-6.

\section{Author contribution statement}

The first three authors of this paper contributed equally to the work. All the others contributed a little less, but importantly, with data acquisition and data analysis.

\section{References}

1. O.V. Penkov, M. Khadem, W.-S. Lim, D.-E. Kim, J. Coat. Technol. Res. 12, 225 (2015)

2. K.-D. Weltmann, Th. von Woedtke, Plasma Phys. Control. Fusion, 59, 012031 (2017)
3. S. Wu, Y. Cao, X. Lu, IEEE Trans. Plasma Sci. 44, 134 (2016)

4. J. Winter, R. Brandenburg, K.-D. Weltmann, Plasma Sources Sci. Technol. 24, 064001 (2015)

5. H.R. Kang, T.H. Chung, H.M. Joh, S.J. Kim, IEEE Trans. Plasma Sci. 45, 691 (2017)

6. T. Abuzairi, M. Okada, S. Bhattacharjee, M. Nagatsu, Appl. Surf. Sci. 390, 489 (2016)

7. C.-T. Liu, K.-Y. Cheng, Zh.-H. Lin, C.-J. Wu, J.-Y. Wu, J.-S. Wu, IEEE Trans. Plasma Sci. 44, 3196 (2016)

8. O. Birer, Appl. Surf. Sci. 354, 420 (2015)

9. T. Wang, B. Yang, X. Chen, X. Wang, C. Yang, J. Liu, Plasma Process. Polym. (early preview)

10. A. Yamamoto, Y. Kuwano, M. Nakai, T. Nakagawa, T. Sakugawa, H. Hosseini, H. Akiyama, IEEE Trans. Plasma Sci. 43, 3451 (2015)

11. S. Wang, J. Zhang, G. Li, D. Wang, Vacuum 101, 317 (2014)

12. H. Nizard, T. Gaudy, A. Toutant, J. Iacono, P. Descamps, P. Leempoel, F. Massines, J. Phys. D: Appl. Phys. 48, 415301 (2015)

13. N. O'Connor, H. Humphreus, S. Daniles, IEEE Trans. Plasma Sci. 42, 756 (2014)

14. Z. Cao, Q. Nie, D.L. Baylist, J.L. Walsh, C.S. Ren, D.Z. Wang, M.G. Kong, Plasma Sources Sci. Technol. 19, $025003(2010)$

15. J. Furmanski, J.Y. Kim, S.-O. Kim, IEEE Trans. Plasma Sci. 39, 2338 (2011)

16. Q.-Q. Fan, M.-Y. Qian, C.-S. Ren, D. Wang, X. Wen, IEEE Trans. Plasma Sci. 40, 1724 (2012)

17. Z. Cao, J.L. Walsh, M.G. Kong, Appl. Phys. Lett. 94, $021501(2009)$

18. M. Ghasemi, P. Olszewski, J.W. Bradley, J.L. Walsh, J. Phys. D: Appl. Phys. 46, 052001 (2013)

19. D.E. Ashpis, M.C. Laun, E.L. Griebeler, National Aeronautics and Space Administration (Glenn Research Center, Cleveland, Ohio, 2012), Tech. Rep. NASA/TM-2012217449, http://ntrs.nasa.gov/archive/nasa/casi.ntrs.nasa. gov/20120009957.pdf

20. M. Holub, Int. J. Appl. Electrom. 39, 81 (2012)

21. M. Machida, Braz. J. Phys. 45, 132 (2015)

22. C. Liu, N. Cui, N.M.D. Brown, B.J. Meenan, Surf. Coat. Tech. 185, 311 (2004)

23. P. Slepička, N. S. Kasálková, E. Stránská, L. Bačáková, V. Švorčík, eXPRESS Polym. Lett. 7, 535 (2013)

24. M. Chaker, M. Moisan, Z. Zakrzewski, Plasma Chem. Plasma Process. 6, 79 (1986)

25. A.B. Sá, C.M. Ferreira, S. Pasquiers, C. Boisse-Laporte, P. Leprince, J. Marec, J. Appl. Phys. 70, 4147 (1991)

26. X. Lu, G.V. Naidis, M. Laroussi, K. Ostrikov, Phys. Rep. 540, 123 (2014)

27. SpecAir software, http://specair-radiation.net/, accessed on: April 2017

28. N. Masoud, K. Martus, M. Figus, K. Becker, Contrib. Plasma Phys. 45, 30 (2005)

29. D. Staack, B. Farouk, A.F. Gutsol, A.A. Fridman, Plasma Sources Sci. Technol. 15, 818 (2006)

30. P.J. Bruggeman, N. Sadeghi, D.C. Schram, V. Linss, Plasma Sources Sci. Technol. 23, 023001 (2014)

31. F. Nascimento, M. Machida, M.A. Canesqui, S.A. Moshkalev, IEEE Trans. Plasma Sci. 45, 346 (2017) 